\title{
Reconfigurable optical-radio wireless networks: Meeting the most stringent requirements of future communication systems
}

\begin{tabular}{|r|l|}
\hline Journal: & Transactions on Emerging Telecommunications Technologies \\
\hline Manuscript ID & ETT-18-0109.R3 \\
\hline Wiley - Manuscript type: & Special Issue Article \\
\hline Duthor: & $29-$ Oct-2018 \\
\hline Complete List of Authors: & $\begin{array}{l}\text { Saud, Muhammad Saad; oulun yliopisto Tieto- ja sähkötekniikan } \\
\text { tiedekunta, Centre for wireless communications } \\
\text { ahmed, iqrar; Oulun Yliopisto Tieto- ja sähkötekniikan tiedekunta, Centre } \\
\text { for wireless communications } \\
\text { Kumpuniemi, Timo; oulun yliopisto Tieto- ja sähkötekniikan tiedekunta, } \\
\text { Centre for wireless communications } \\
\text { Katz, Marcos; oulun yliopisto Tieto- ja sähkötekniikan tiedekunta, Centre } \\
\text { for wireless communications }\end{array}$ \\
\hline Keywords: & $\begin{array}{l}\text { optical wireless communications, visible light communications, universal } \\
\text { software radio peripheral, reconfigurable wireless networks, software } \\
\text { defined networks, GMSK modulation }\end{array}$ \\
\hline $\begin{array}{l}\text { Note: The following files were submitted by the author for peer review, but cannot be converted to PDF. } \\
\text { You must view these files (e.g. }\end{array}$ \\
\hline movies) online.
\end{tabular}

\section{SCHOLARONE ${ }^{\text {IM }}$ \\ Manuscripts}


DOI: $\mathrm{xxx} / \mathrm{xxxx}$

\title{
Reconfigurable optical-radio wireless networks: Meeting the most stringent requirements of future communication systems
}

\author{
Muhammad Saad Saud I Iqrar Ahmed I Timo Kumpuniemi I Marcos Katz*
}

Centre for Wireless Communications, University of Oulu, Oulu, Finland

\section{Correspondence}

Marcos Katz. Email: marcos.katz@oulu.fi

Present Address

Pentti Kaiteran katu 1, 90014 Oulu. Finland.

\section{1 | INTRODUCTION}

Development of wireless networks has always been driven by the need to enhance communication performance, with particular focus on the supported data rate at link level as well as capacity at system level. These goals clearly stem from the popularity of services and applications consuming large amounts of bandwidth as well as the rapidly increasingly number of wireless devices worldwide. As the number of mobile devices surges and the need for higher bandwidth increases, other issues become

\section{KEYWORDS:}

optical wireless communications, visible light communications, universal software radio peripheral, reconfigurable wireless networks, software defined networks, GMSK modulation, HetNet, testbed.

\begin{abstract}
mary
In this paper, we propose a reconfigurable optical-radio wireless network characterized by high flexibility and performance. The hybrid network can be dynamically configured, adapting itself to the changing requirements of users or operating environments, dynamics of the transmission medium and availability of resources. First, the basic concept, system architecture and key operating modes are introduced. Possible operating scenarios and their relationship to the operating modes are discussed. network components, architecture, modulation scheme, and implementation technologies. Implementation was carried out using mostly off-the-shelf components and universal software radio peripheral (USRP) blocks. Particular attention has been put in implementing a fast handover mechanism between modes, an essential requirement for seamless network reconfiguration. Network performance is evaluated and different switching approaches are compared. The paper demonstrates practically the proposed concept based on dynamic system reconfiguration. For the implementation, a mode switching algorithm was developed making decisions based on the quality ted through the network. System performance was assessed through data throughput and packet loss measured for different modulation parameters. The proposed concept, essentially a software defined hybrid network, is flexible and makes efficient use of radio resources. Moreover, the network has the potential to achieve high performance. All these characteristics make reconfigurable optical-radio networks an attractive candidate for fifth generation $(5 \mathrm{G})$ and beyond.
\end{abstract}


highly relevant, in particular the overall price to be paid to achieve this level of connectivity. Thus, design of spectrum, energy and cost-efficient wireless networks has also become one of the key goals of modern communications, with a direct impact on every stakeholder of the communication chain. Depending on the scenarios and applications, other important requirements for wireless networks include low latency, high reliability, security and privacy. Complying with many of these requirements is one of the most important challenges of the communication engineer, due to multiple design tradeoffs, in particular the compromise existing between performance complexity and usage of available radio resources.

Wireless communication has traditionally been based on radio technology. This trend continues, and today the overwhelming majority of wireless communications exploit radio waves. Indeed, radio frequency (RF) communications have become an integral part of our life but the rapidly increasing number of devices entering the network, which could be in billions by 2020 , is leading towards a spectrum crunch [1]. This has motivated research in alternative transmission technologies, such as wireless communications exploiting light. Indeed recent advancement in optoelectronics, particularly the development and further improvement of white light emitting diodes (LED), have created an attractive approach to send information wirelessly, namely optical wireless communication (OWC). Visible light communication (VLC) exploits the lighting infrastructure to create wireless communication links through optical medium. The initial model of OWC was demonstrated by Kahn and Barry [2] in 1997, despite the channel degradation challenge the authors demonstrated OWC using infrared (IR) an efficient mean of communication for short range applications. In recent years, there has been a considerable interest in developing VLC technology, motivated by its unique advantages, in terms of its huge and unregulated bandwidth, inherent security and privacy, no radio exposure, among others. In terms of short-range communications, VLC can be seen as complementing radio communication, rather than competing with it. This is an important observation, as these two technologies could be exploited in a complementary fashion, aiming at combining the advantages of both. Even though there is considerable amount of research and development on VLC technology over the past 15 years, the technology has not made a market breakthrough yet, remaining mostly in research laboratories. One of the reasons for this is the fact that VLC has been commonly regarded as an isolated communication technology. In a realistic context, the dominant role of the radio communications cannot be ignored. Indeed, the current role of radio in the wireless world is unquestionable, and radio will continue to be the key technology in the future as well. However, optical wireless technologies are expected to increasingly find their place, as radio spectrum is rapidly being occupied. Exploiting the complementarity of radio and optical wireless networks will not only help to fulfill the most stringent requirements of future wireless networks but it will also position VLC as a mainstream technology on its own. As discussed in [3], future wireless communication networks will be based on multiple access technologies, the pervasively available radio as well as optical wireless communications. The ever increasing pressure created by growing spectrum usage and performance requirements is pushing the wireless community to consider optical-radio networks as a promising alternative for future communication systems. In this paper, a flexible and highly integrated optical-radio wireless network is presented and discussed as a high-performance flexible solution to the challenges of future wireless networks.

The proposed reconfigurable optical-radio network can be seen as a software defined hybrid network (SDHN). Figure 1 shows the concept of SDHN in practice. Equipment such as mobile devices, home and office appliances and any possible internet of things (IOT) node are equipped with optical-radio transceivers (ORT). Also lighting infrastructure has integrated ORTs. Devices and objects are connected using the most appropriate technology, which, depending on channels conditions and changing requirements, could change with time. Consider the simple example where a user enters a sensitive environment, such as an aircraft, a hospital or a manufacturing plant. The system (e.g., host building, network operator) will switch off radio transceivers of the user device and switch on optical interfaces, creating a radio-free environment. From a security and safety points of view, VLC is worthwhile in aircrafts and hospitals, as suggested in [4, 5]. A mobile device in a user pocket, i.e., unable to work in optical mode, will be connected via radio, but once it is removed from the pocket and exposed to light, the optical link could be used. In a spectrum-congested scenario (e.g., convention center, etc.) the operator could use optical links to off-load to use resources in a more balanced fashion.

Vertical handover (VHO) between radio and optical wireless networks has been studied in the past, mostly from a theoretical perspective, where typically different switching criteria have been investigated [6, 7]. Network handover is necessary to provide secure, safe and uninterrupted service, for instance, due to different coverage areas of the networks, sudden signal blockage, requirements of the scenario, decision of network operators or users, and others. To be practically attractive, handovers should be transparent to the user and the application being used. This requires an implementation that guarantees a fast switching mechanism i.e., seamless handover. This is an essential requirement for a flexible hybrid network solution presented in this paper. This paper focuses on the vertical handover problem, and how to reduce the switching time. This is tightly related to system architecture, particularly the OSI layers involved in the switching process as well as on the actual implementation of the 
3

4

5

6

7

8

9

10

11

12

13

14

15

16

17

18

19

20

21

22

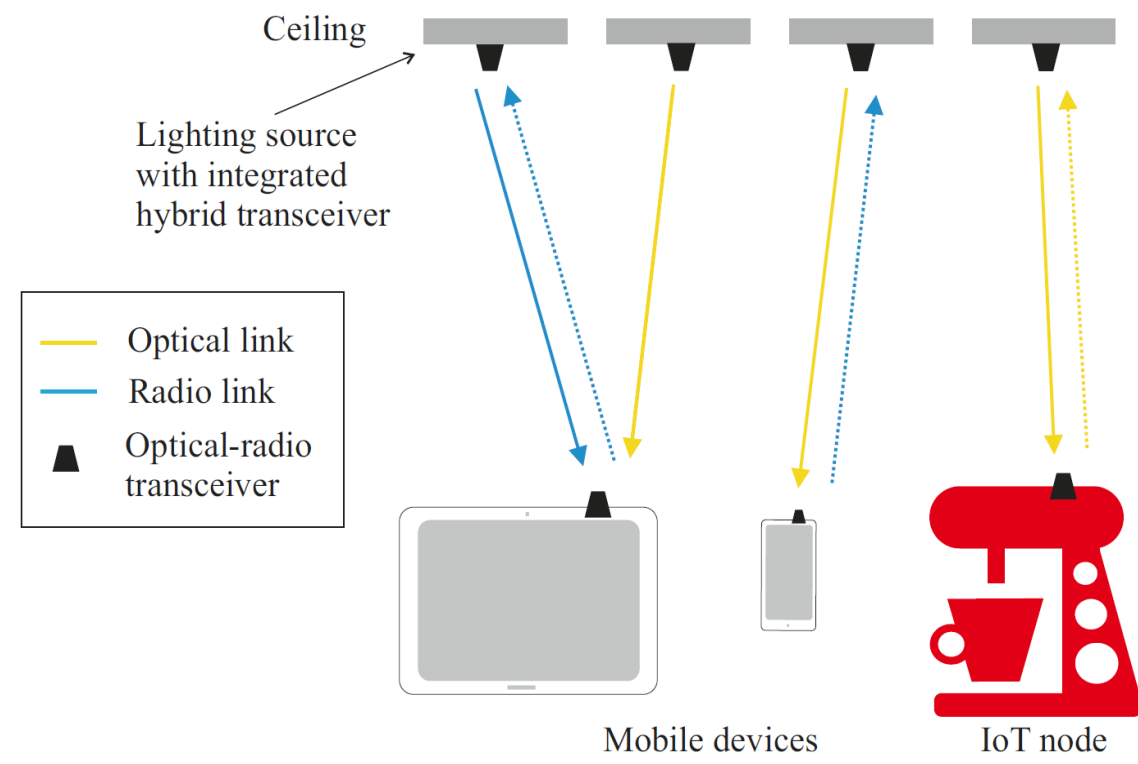

FIGURE 1 Concept of a SDHN in practice: Mobile devices and IoT nodes can be connected using optical and radio links.

system. Thus, the implementation of fast handover between optical and radio networks is highly relevant in order to have highly responsive wireless communications network.

A number of recent publications have considered practical implementations of hybrid networks involving VLC technology. A hybrid power line and visible light communication system is studied in [8] in the context of e-health management. The modulated signals for the LED are generated by a power line communications (PLC) device, designed by the authors, exploiting thus the existing power and lighting resources, as well as establishing communications where RF is restricted. VLC is implemented using software defined radio (SDR) in [9], where the SDR devices can adapt dynamically. These devices give freedom of testing different signal processing and modulation schemes. Authors considered various modulation schemes including OFDM, Minimum Shift Keying (MSK) and Phase Shift Keying (PSK) for video streaming while evaluating their system. VLC has proven to be beneficial and data rates from several Mbits/s to few Gbits/s for indoor communication have been tested [10. 11. 12]. Very few studies have been conducted where VLC and RF are jointly used, though such research could help to better exploit the synergy between these transmission technologies. To demonstrate the performance of VLC, a comparison for throughputdistance model is drawn between a hybrid optical-radio wireless network and a VLC hotspot in [13]. The throughput comparison for single and multiple users reflects that hybrid optical-radio networks yields lower outages. In [14] a novel way of using RF for full duplex and VLC for downlink is presented. The RF full duplex is used to provide the communication when the VLC downlink deteriorates. Authors evaluated the performance of their algorithm for multiple users where handover between VLC hotspots as well as between VLC and RF were also evaluated. In another study conducted for a hybrid optical-radio network operating in indoor environments, authors demonstrated how efficiently a VLC can be embedded to an existing RF network [15]. Authors discussed three working scenarios, that include only Wi-Fi communications, downlink streaming via VLC and VLC and Wi-Fi working in parallel. When using a single VLC link and Wi-Fi, maximum throughput is obtained when 6-7 out of 10 users are using VLC as downlink and the remaining are on Wi-Fi. Results show, as expected, that the throughput for the hybrid system is better than that for standalone VLC or RF system. Handovers have a critical role in hybrid systems, and in [6], a fuzzy logic based algorithm for making handover decision in hybrid system is proposed and evaluated. The algorithm makes decisions on fuzzy rules that are proposed using probabilistic counts of interruption and failed messages. In our previous work [16, 17], a hybrid optical-radio network capable of switching between VLC and RF at very high speed was implemented. We tested it for single user and evaluated the testbed by capturing a video stream with very low latency. Hybrid optical-radio networks are expected to become widespread, as preliminary results show a great potential in a number of areas. Exploiting VLC and radio networks in a flexible, quickly reconfigurable fashion, as suggested here, will allow to achieve high performance while reduce the overburdening of radio spectrum, while enhancing security and safety of users. 
This paper is organized as follows. Section 2 introduces a novel architecture for a reconfigurable optical-radio wireless network, supporting fast handovers. Different operating modes are described, and a number of possible scenarios particularly attractive for the network are described. Section 3 considers the practical implementation of the proposed concept, vertical handover problem across the optical-radio network domains. Technical solutions that are investigated and implemented, including modulation scheme and a performance comparison based on measurements. Section 4 concludes this paper.

\section{2 | TOWARDS A FLEXIBLE HIGH-PERFORMANCE RECONFIGURABLE OPTICAL-RADIO WIRELESS NETWORK}

This section discusses in more detail the proposed concept of a reconfigurable optical-radio wireless network. The network conveys information in both directions, exploiting the optical and radio mediums. Reconfiguration means here that according to a number of parameters reflecting the state of the physical medium, user and service requirements or local operating policies. The network is dynamically configured to use in each direction of transmission either by optical or radio links. Different operation modes and possible operating scenarios are also described in this section.

\section{1 | System Architecture}

The basic architecture of the reconfigurable network is depicted in Figure 2. The baseband processing block contains all the communication functions required in the transmitting and receiving chains. The optical and radio front ends are the interfaces to the respective physical medium. Note, that in this case optical and radio links use their own chains, allowing the implementation of particular communication functionalities in each chain, e.g., modulation and coding schemes. This approach also allows the simultaneous use of both links, resulting in the possibility of implementing a cooperative/diversity mode where the optical

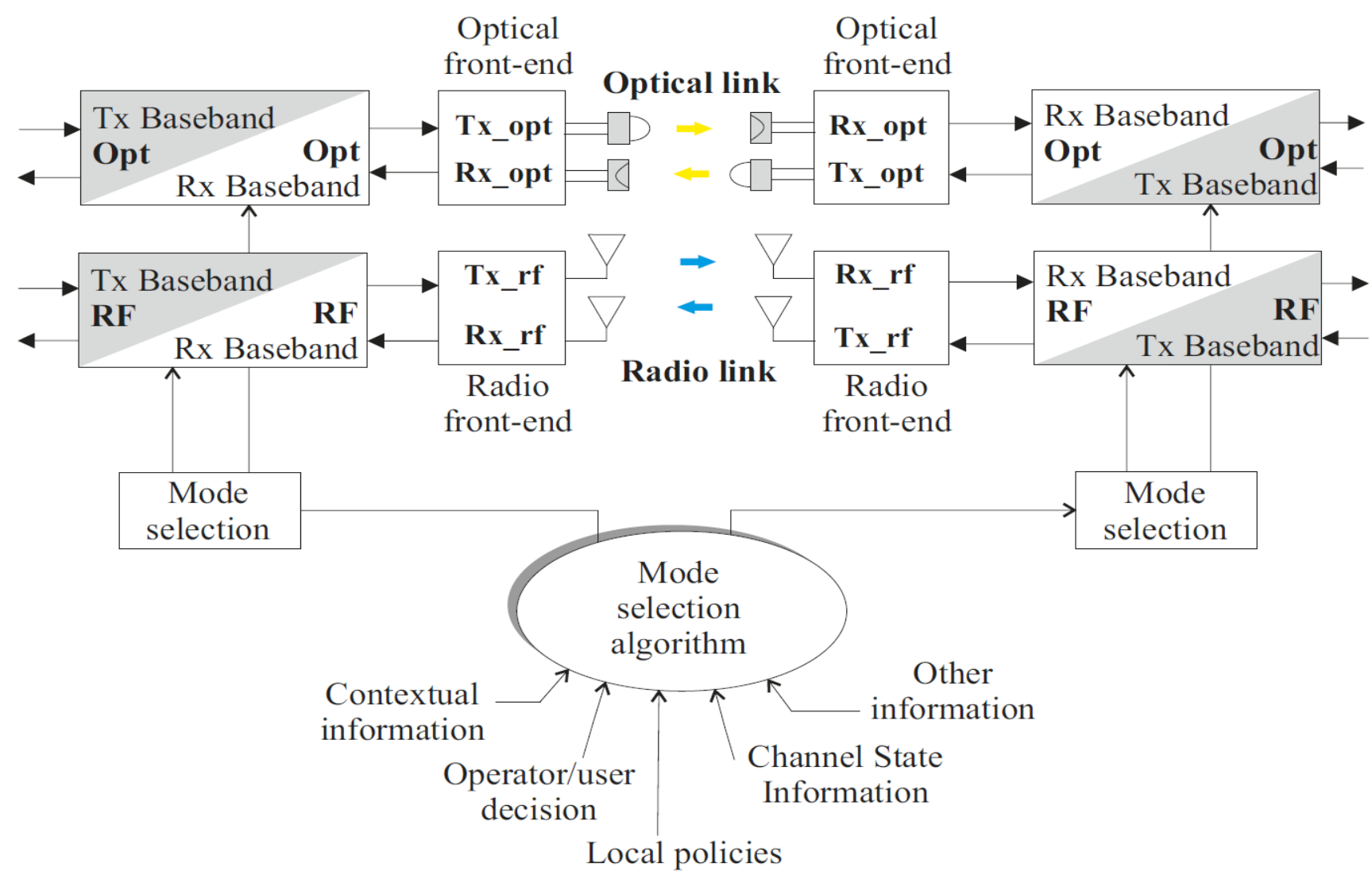

FIGURE 2 Basic architecture of a reconfigurable optical-radio wireless network. 
and radio channels are concurrently exploited. In principle, the network can also be implemented with a single reconfigurable transceiver on each end, though this solution is less flexible than the here considered. The system in Figure 2 is controlled by a mode selection algorithm, which processes different information and decides how to configure the network. The decisionmaking process for reconfiguration receives input information such as a) channels state from the optical and radio mediums, b) contextual information such as type of environment, local usage policies and regulations, etc., c) user and network operator decisions, d) current usage of available resources, and others. Note that this architecture allows reconfiguration at both, shortand long-term scales. The former refers to reconfigurations following rapid fluctuations of channels and other instantaneous events, whereas the latter refers to more lasting selections, for instance based on the type of environment a wireless device is operating. Certainly, both approaches can be readily combined.

\section{2 | Modes and Mode Selection}

There are basically five operating modes in which the system can be configured to, depending on how optical and radio chains are selected. The system can be configured in Basic Modes, Hybrid Modes as well as a Cooperative Mode. Figure 3 depicts the operating modes. Basic Mode refers to fully-optical and fully-radio cases, corresponding to Mode 1 and 4 in Figure 3. Hybrid Mode combines optical and radio links, as optical/radio for downlink/uplink (Mode 2) and radio/optical for downlink/uplink (Mode 3). Finally, Mode 5 considers the case where both optical and radio links are simultaneously used. The algorithm controlling the mode selection could in principle consider different input parameters in order to make a decision. In its simplest form, the algorithm can be defined as a rule or set of rules mapping a number of situations, events or measurements with their associated modes. More complex approaches can also be developed, where the mode selection depends on number of parameters that can be weighted according to their relevance. Operating modes are also highly related to the operating scenario the hybrid network is working in. A number of attractive scenarios of the considered network is presented next.



FIGURE 3 Operating modes of a reconfigurable optical-radio network.

\section{3 | Operating Scenarios for Optical-Radio Wireless Networks}

Even though the hybrid optical-radio network can be used virtually in any practical scenario, there are some particular scenarios where this concept is greatly appropriated. Here we briefly present a few scenarios and discuss how the switching algorithm could be devised to match the requirements of these scenarios.

- Interference-sensitive environments: This is a highly relevant scenario, where interference is a key issue e.g., environments where sensitive equipment are in use. This is the case of hospitals, manufacturing plants and aircrafts, among others. Maximizing the use of optical communications while minimizing the use of radio would be a relevant strategy in these cases. Note that only optical (Mode 1) would be the ideal operating mode in sensitive environments, but the nature 
of optical communications (e.g., light prone to be blocked, uneven coverage, etc.) requires the involvement of radio transmissions whenever needed. One approach for designing the switching algorithm would be: use optical communications as the default technology, and switch to radio communications whenever required (e.g., sudden optical shadowing).

- Security/privacy/safety: In some use cases, secure communications is of utmost importance, as in hospitals, local tactical communications, corporate communications and others. Again, in these scenarios, the inherent confined propagation of optical wireless communications makes this option the preferred operating mode whenever possible, involving radio communications sporadically. As in the previous case, the switching algorithm could be designed to maximize the use of Mode 1. In addition, by maximizing the use of Mode 1 the amount of radio exposure is minimized, resulting in a safe wireless communication system.

- High performance: In some situations, support of very high speeds might be needed. This could be readily supported by using Mode 5, where both optical and radio links are used in parallel.

- High reliability: Reliability, needed in mission critical communications, tactical communications and also in certain applications in medical field where it can be implemented with the proposed concept. This can be achieved by Mode 5 by configuring it to exploit diversity. One approach could be to use selection diversity, selecting always the air interface with best associated channel.

- Off-loading: VLC technology exploits a vast and unregulated zone of the spectrum, several orders of magnitude larger than the spectrum allocated to radio communications. Even in a conventional only-radio operation, Mode 4, other modes could be activated to allow for additional bandwidth whenever radio spectrum is congested.

\subsection{Importance of Energy Efficient System}

Energy efficiency is an increasingly important concept in all sectors of our life due to environmental, ethical and cost reasons. Several aspects of energy efficiency have also been considered in wireless communications. The size of the electronic devices is constantly decreasing while computing power and capacity are increasing. This has lead to the development of various kinds of sensors and sensors networks that can be deployed in a variety of environments. Often the sensors are battery operated and/or the number of sensors is large leading to a need to minimize the power consumption. The rapidly increasing number of applications in the field of IoT is an example of this trend. Currently, there is a great drive in the development of the 5G mobile communication systems including IoT as one of its vertical fields, thus speeding up the needs for the energy efficiency from this direction as well.

Energy efficiency has an equally important role in RF based systems as well as in the VLC systems. VLC is often reported to have an inherently high energy efficiency and run on power from grid. However, VLC devices may also be battery powered and/or they may operate in environments where general lighting is not necessary or even allowed.

Energy efficiency is also taken into account for hybrid network studied in this paper. The power amplifiers (PA) both in the RF and VLC links must be operated as efficiently as possible to minimize energy expenditure i.e., the operation point of the PAs must be set close to the one decibel compression point providing still linear operation with a high efficiency. By this means, the signal constellation will not be deteriorated and the signal spectrum will not give rise to an increase in the adjacent channel power ratio due to signal spectrum smearing [18]. These demands can be satisfied with the usage of a constant envelope modulation method as, e.g., Gaussian Minimum Shift Keying (GMSK), which is utilized in our testbed both in the VLC and RF link.

The testbed under discussion in this article has several possible operation modes. In some of the modes VLC is selected as the default communication method with RF remaining as a supportive method, e.g., when the VLC link be broken. In this case, the RF link is set to a sleep mode. In order to monitor the possible need for it to be taken into operation it must poll the requests from the testbed. Slower polling frequency causes an increase in the switching delay and higher polling frequency causes an increase in the power consumption, respectively. A solution to achieve constant monitoring with low power consumption would be the adoption of wake-up radios in the RF link as proposed in [19]. The implementation of wake-up radios in the testbed is one of the future work topics in our development work. 


\section{3 | PRACTICAL IMPLEMENTATION OF A RECONFIGURABLE OPTICAL-RADIO NETWORK}

In this section, we discuss the practical implementation of an experimental reconfigurable optical-radio network. The considered network architecture and the employed modulation scheme are first introduced. A fundamental requirement to implement this concept is the capability of the system to perform quick handovers (i.e., mode switching). The used handover approach is also explained in this section. When discussing about handovers, one should consider the time scale at which they can be executed and the switching time associated with the operating modes.

\section{1 | Basic Hybrid Network Architecture}

We present the experimental setup of a hybrid optical-radio network, implemented based on the architecture described in section 2 , as an extension of our previous work [16, 17]. The system is capable of dynamically switching between the networks, according to a switching algorithm. The experimental network is based on a relatively simple implementation, using as much as possible off-the-shelf components. The system was not optimized to attain high data throughput but for achieving fast handover between modes. The VLC downlink is established using a $24 \mathrm{~W}$, commercial (Philips) ceiling LED luminaire [20] whereas the VLC uplink uses an IR LED, using BIAS-T configurations [21,22]. We exploited the dynamic reconfigurability properties of universal serial radio peripherals (USRPs) [23] to implement the hybrid network. The data is transmitted to and received from the USRPs through Ethernet, which is configured in such a way that the communication between both USRPs is continuously monitored. Different algorithms can be implemented, based on number of possible switching criteria in the experimental system to test the switching capabilities. The implemented algorithm maximizes the use of the optical links, reducing the amount of generated radio interference. This strategy is attractive in radio-sensitive environments. In case of a breakage of the physical link of VLC (e.g., light obstruction), the switching algorithm switches to the radio network and starts sending the information on the radio link. Four NI 2920 USRPs have been used to establish VLC and RF links between source and sink. The architecture is depicted in Figure 4. The system architecture supports uninterrupted point-to-point communications from source to sink and vice versa (i.e., uplink and downlink). The architecture is developed based on User Datagram Protocol (UDP) transmission, the data at source end is sent simultaneously to two USRPs, one USRP implements source node for VLC while the other USRP is for RF source node. The remaining two USRPs connected to the sink end realizing the sink nodes for VLC and RF.

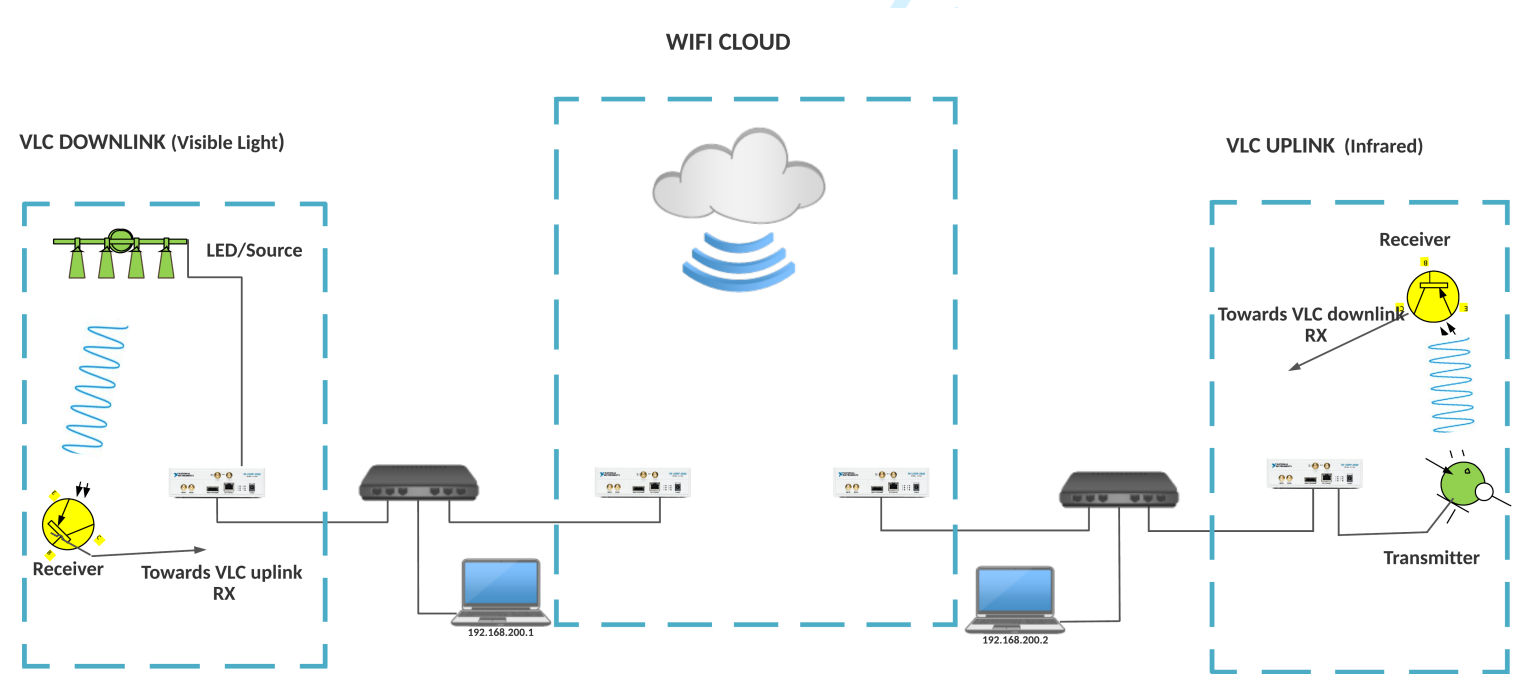

FIGURE 4 Simplified working layout of the implemented hybrid optical-radio network. 


\section{2 | Modulation Schemes}

The hybrid VLC testbed under investigation uses GMSK modulation format [18, 24] both in the optical and RF links. GMSK is an extension of the full response modulation scheme [18, 25] MSK [18]. Both modulation approaches are constant envelope modulation schemes [26]. GMSK can be generated by filtering the input bit stream with a Gaussian filter prior to the MSK modulation, as shown in Figure 5. The MSK modulator can be of any structure, as described in [27], or just a simple frequency modulated modulator, as presented in [24]. The transfer function of the Gaussian filter can be stated as

$$
H(f)=\exp \left(f / B_{b}\right)^{2} \ln 2 / 2,
$$

where $f$ is the frequency and $B_{b}$ is the $-3 \mathrm{~dB}$ bandwidth of the filter [28]. One key parameter to describe the GMSK-modulation is the time-bandwidth product (BT) of the Gaussian filtering process, which is the multiplication of the bit time and $B_{b}$. The Gaussian filtering has the effect of changing the full-response MSK signal into a partial response signal. It also modifies the signal spectrum to be more compact providing lower out-of-band radiation. The lower the BT product, the more compact spectrum is achieved. On the other hand, with small BT values, the GMSK signal deviates more from the full response MSK signal when investigated, e.g., in a trellis diagram. The selected modulation scheme has a substantial effect on the operation and performance of the network. Modulations such as, e.g., quadrature phase shift keying (QPSK) did not perform satisfactorily in the optical link. This can be explained by the slowness of the (phosphorous-based) LEDs, that are not able to follow the abrupt phase changes in the driving QPSK signal, thus degrading the constellation diagram of the modulation. By using a constant envelope modulation method this problem can be avoided as the signal changes smoothly between symbols. The BT value of modulation has also a significant effect on the performance, as noticed in [29], where GMSK with several BT values was compared to MSK both through simulations and measurements in a VLC short-range communication case. Authors showed in [29] that the selection of the BT value has a clear impact on the achievable bit error rate of the VLC link. In the experimental testbed BT was varied from 0.35 to 0.7 . The selection of a proper GMSK BT value is an important topic to achieve a good bit error rate performance and enough spectrum compactness, providing low out-of-band radiation at the same time.

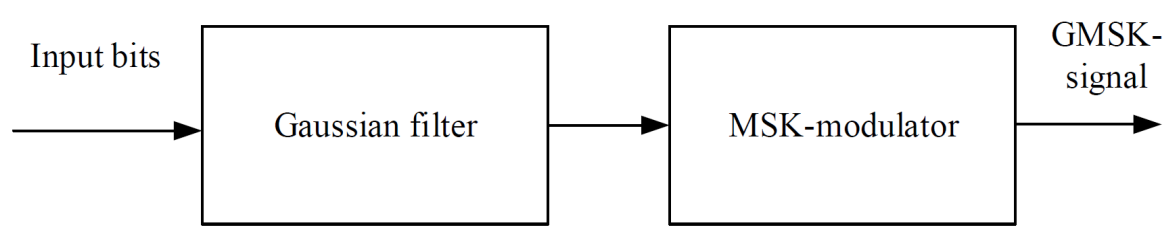

FIGURE 5 Block diagram of a GMSK modulator.

\subsection{Experimental Setup for Implementing Vertical Handover}

The goal was to design a hybrid system that can transmit data seamlessly while switching between different networks. The switching/handover could be rules-based and our aim is to design a system that can dynamically adopt the predefined handover rules to select the best mode. These rules could be based on link failures, direct user decision or might depend on local policies (e.g., in radio-sensitive scenarios VLC will be preferred, as mentioned), keeping high degree of flexibility is key since we are interested in using this network in different working environments. Our implemented system is capable to switch between the aforementioned five different modes and switching can be dynamic, fixed or time dependent based on predefined rules implemented in the switching algorithm. Usually fixed or time dependent switching is under the influence of local policies and is for user's ease who can change the operational mode sensing the environmental e.g., in radio sensitive areas or in populated places. Other rules could be depending on user population or data load over the network. We devised an immediate vertical handover (VHO) strategy for the implemented system. Our default mode is 5, the system establishes both VLC and RF tunnels when started. The data by default is transferred through VLC tunnel, the RF tunnel is idle at this point. If interruption occurs the VHO switches to the RF link and start sending data through RF tunnel. The user can switch to any other mode deciding on 
local policy for link to be used.

In order to adapt the changes automatically, we have proposed some rules based of fuzzy logic (FL) to make the switching decision. Since our system is in principle designed to work in cooperative mode where both RF and VLC uplink and downlink are established when the system is turned ON, then we monitor both uplink and downlink for VHO. For adaptation or automatic switching between modes, we make use of interruption to construct a fuzzy set. The set can then be used for further decisionmaking. The interruptions are counted as short i.e., $T_{s}<k$ seconds and long $T_{s}>k$ seconds, where $\mathrm{k}$ is an amount of time that can be defined by the designer. The fuzzy set records some interruption first, for the next interruption the system estimates its probability of being short or long and takes one of the following decision.

Decision 1: If the probability of next interruption of becoming either short or long interruption is more than $75 \%$ on both downlink and uplink sides, then Mode 4 is selected.

Decision 2: If the probability of next interruption of becoming either short or long interruptions on downlink side is more than $75 \%$, then Mode 2 is selected.

Decision 3: If the probability of next interruption of becoming either short or long interruptions on uplink side is more than 75 $\%$, then Mode 3 is selected.

As mentioned above for $\mathrm{VHO}$ and adaptation, we use link monitoring information information. We implemented the universal TCP/IP suit for packet encapsulation, where the bottom two TCP/IP layers can provide link information. One option is to use link information provided from these layers, since the implementation uses Linux tunnel (TUN) driver [30] and it does not take any link information from the data link layer. Therefore both medium access control (MAC) and logical link control (LLC) sublayers of data link layer are idle in our case, which is why we omitted both. For the dynamic handover in Mode 5, we incorporated a strategy in our algorithm that monitors the link state. For this reason we added a sublayer below the network layer to the TCP/IP stack. This makes the switching fast; our algorithm sends protocol packets on VLC PHY for checking the VLC link state. We added an exponential delay to avoid continuous VLC link monitoring, which means that if the VLC link is not restored, then protocol packets would be resent after certain time. Meanwhile, the RF network is used for communication. Figure 6 shows the TCP/IP stack. Note that the new switching sublayer, added below network layer, performs all the switching of network in case of link failure. Data link layer in our algorithm is not used; packets are transmitted and received directly from physical layer.



FIGURE 6 Modified TCP/IP stack.

Both VLC and RF links are established using the GNU radio package [31]. The GNU radio software creates a tunnel between the computer at sink or source end and the USRP in order to transmit or receive data packets. The Linux tunnel (TUN) driver creates a virtual buffer in the source and process data packets in it. These packets are then transferred to either source or sink node. The TUN driver has two virtual sublayers MAC and PHY. The MAC sublayer receives the packets from source and sends them to PHY sublayer connected to USPR, and vice versa. Figure 7 shows the packet control flowchart. RF link is up all the time while the VLC link is default and is responsible for streaming. 


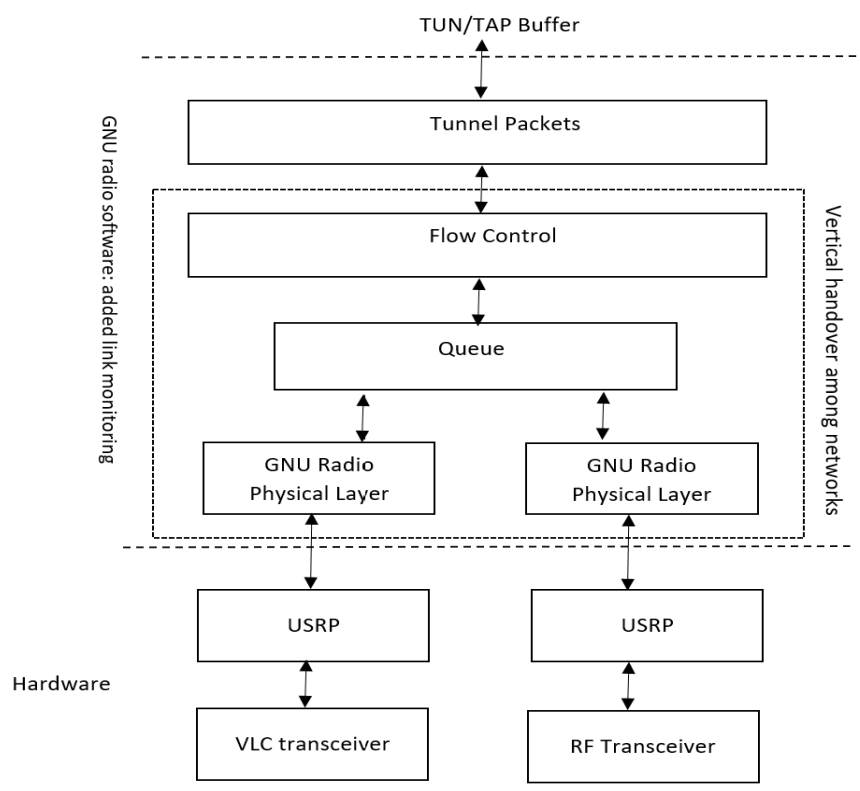

FIGURE 7 Packet control flow chart.

The GNU radio program starts by initiating the main thread that generates RF and VLC blocks. These blocks contain the relevant information such as modulation, demodulation schemes, receiver and transmitter frequencies, gains, bit rates, etc. The software handles the packets using number of other threads. VLC is the default link when using the cooperative mode. After initiating the main thread, the software initializes the Rx and Tx queues and TUN accepts the packet by reading the tunnel file descriptor. Then, it checks the VLC link state, if the link is up the packet is then transferred to VLC PHY layer. The relevant link information is added to the packet before the software handovers it to the VLC PHY layer. The information added to the header contains the Rx link state and few bytes for distinguishing actual or dummy packets. Otherwise, the packet goes to the Tx queue. Once the packet is sent or received the corresponding timing is registered. This time is then subtracted from the time of previous transmitted or received packet to get the Tx and Rx delays. To monitor VLC, a thread named secondary is initiated parallel to main thread. We set thresholds for Tx and Rx and compared estimated delays with thresholds. When the Tx delay is greater than the threshold, the software starts sending dummy packets. The dummy packets include few bytes representing the dummy information ( 2 bytes), the Tx link information ( 2 bytes) and the payload (as zeros, 36 bytes). A small delay is added for monitoring the link to avoid overhead. If the VLC is up, the main thread will transmit packets through VLC Tx and VLC PHY would receive the packets at the other end. Otherwise, the main thread will send packets to Tx queue. The secondary thread will switch the network from VLC to RF and RF thread will start sending the packets using RF Tx. To receive the packets from RF PHY another thread named Rx queue will start accepting packets coming RF PHY, which are then available to the user, e.g., as video, audio etc.

\section{4 | Channel Modelling}

Low cost and easy to install attributes of LEDs make them more suitable for OWC in indoor scenarios. Luminance of minimum $400 \mathrm{~lx}$ approximately or above is necessary, which is well within the illuminance requirements of working spaces within organizations as set by International Organization for Standardization (ISO) [32]. For our study, a single 21 x $21 \mathrm{~cm}$ commercial Philips white led module, thus we will examine the square illumination configuration. Table 1 shows the light characteristics parameters, these statistics are then used to estimate different characteristics such as impulse response, signal-to-noise ratio (SNR) etc. The receiver is placed at desk height i.e., $0.85 \mathrm{~m}$ whereas the distance between source and receiver is $1 \mathrm{~m}$. We are interested in line of sight (LOS) communication; the measured illumination pattern can be seen in Figure 8.

From the Figure 8, it is clear that maximum intensity is obtained right under the VLC hotspot and it falls off when towards the edges of illuminance area. The impulse response for LOS channel is usually a single peak with a certain short delay caused due to the propagation delay of the light traveling from source to detector. Received power or intensity values are measured 




FIGURE 8 Measured illumination pattern for the geometry of the implemented hybrid network over $1 \mathrm{~m} x 1 \mathrm{~m}$ area.

by placing a photodiode receiver at different places in a $1 \mathrm{~m}^{2}$ area. Table 2 gives the details on received optical power in LOS. As mentioned, the maximum optical power is concentrated in the middle and standard deviation of 0.11 mw shows the optical power fall off towards the edges.

TABLE 1 LED Source characterisitcs.

\begin{tabular}{ll}
\hline Active area & $21 \mathrm{~cm}^{2}$ \\
Refractive index & 1.5 \\
Semi angle at half power & 35 \\
Number of LED & 12 \\
FOV & $60^{\circ}$ \\
\hline
\end{tabular}

TABLE 2 Received power [mW].

\begin{tabular}{ll}
\hline Maximum Power & 0.37 \\
Minimum Power & 0.14 \\
Average Power & 0.25 \\
STD & 0.11 \\
\hline
\end{tabular}

Generally, multipath and optical path losses are considered when modelling the optical channels. Since we are dealing with LOS communication, multipath losses are not considered in our case. In LOS communication, maximum intensity segments are transmitted via direct channel and intensity pattern is modelled considering by Lambertian illumination. For a Lambertian illumination the received optical power contains both LOS and diffused segments, which can be expressed as

$$
P_{r}=\sum_{i}^{n} H_{i}^{l e d}(0) d P_{l e d}(t-\tau)+\sum_{i}^{k} H_{k}^{d i f f}(0) d P_{d i f f}(t-\tau),
$$

where $H_{i}^{\text {led }}(0)$ is channel gain, $d P_{l e d}$ is the power of LED and $\tau$ is delay light taken to reach the detector. Neglecting the second term, since we are only taking LOS packets

$$
P_{r}=H_{l e d}(0) d P_{l e d}(t-\tau),
$$


The Lambertian intensity pattern can be expressed mathematically as.

$$
L_{o}(\phi)=\left\{\begin{array}{lc}
\frac{m+1}{2 \pi} \cos ^{m}(\phi) & -\pi / 2 \leq \phi \leq \pi / 2 \\
0 & \phi \geq \pi / 2,
\end{array}\right.
$$

where $m$ depicts order of Lambertian emission, and $\phi$ is irradiance angle. Since we are only taking LOS, $\phi$ is 0 . The order of the $m t h$ Lambertian emission $m$ can be estimated as

$$
m=\frac{\ln (2)}{\ln \left(\phi_{1 / 2}\right)}
$$

where $\phi_{1 / 2}$ is semiangle at half power. Thus the gain of the direct or LOS optical channel can be estimated as

$$
H_{l e d}(0)=\frac{m+1}{2 \pi d^{2}} \cos ^{m}(\phi) \cos (\theta) g(\psi) \cdot A_{d e t},
$$

where $A_{d e t}$ is the area of silicon photodetector used, which is $1 \mathrm{~cm}^{2}$ in our case and $\theta$ is the angle between the source and surface normal. $g(\psi)$ is the optical gain of filter if used. The intensity in radiated angle can be expressed as

$$
I_{R}=I_{0} \cos ^{m}(\phi),
$$

where $\phi$ is the irradiance angle, the radiant light is dependent on angle of emission. The impulse response and transfer function of the system can be expressed as

$$
\begin{gathered}
h(t)=\frac{m+1}{2 \pi d^{2}} \cos ^{m}(\phi) \cos (\theta) g(\psi) \cdot A_{d e t} \delta(t-\tau), \\
H(f)=\frac{m+1}{2 \pi d^{2}} \cos ^{m}(\phi) \cos (\theta) g(\psi) \cdot A_{d e t} \exp -j 2 \pi \tau,
\end{gathered}
$$

where $\tau=d / c$ is the propagation delay light suffers in transmission and $\mathrm{c}$ is speed of light. The SNR of the system can be calculated with following equation

$$
S N R=\frac{\left(P_{r} \gamma\right)^{2}}{2 q \gamma\left(P_{r}+P_{n}\right) B+\sigma B},
$$

where $P_{r}$ is received power, $\gamma$ is detector sensitivity. We assumed the sunlight ambient noise as background noise having value $P_{n}=43 \mathrm{nW} / \mathrm{m} 2 / \mathrm{Sr} / \mathrm{nm}$ and $\sigma=5 * 10^{-12} \mathrm{~A} / \mathrm{Hz}$ is the amplifier noise. We estimated SNR at different data rates [33]. Since we have taken LOS intensity values so we left out the power received due to reflections.

\section{5 | Handover Performance}

As mentioned in section 3.3, we designed a fast vertical handover for our reconfigurable wireless network, ensuring seamless communication, for instance when the VLC link goes down or restores. We set VLC as default link for performance evaluation. For VLC links, tunnels of 3.125 and 6.25 Mbps are created between source and sink nodes, all using GMSK modulation. While for RF, we set frequencies of $425 \mathrm{MHz}$ for downlink and $325 \mathrm{MHz}$ for uplink. The five modes can either be used dynamically or based on user requirement or local policies e.g., in closed spaces having visible light such as hospitals, offices or planes etc. will prefer Mode 1. Mode 2 can be used during night, while Mode 3 and 4 can be best for sharing users load. The software compares Tx and Rx delays with thresholds set to $300 \mathrm{~ms}$ and $600 \mathrm{~ms}$, respectively. The handover time is measured to be $877 \mathrm{~ms}$. Packet loss could be reduced by sending packets though buffer. Moreover, we measured a latency of two seconds when streaming the video between two nodes.

Table 3 presents the performance metrics of our optical-radio network. We estimated the packet loss for three time-bandwidth values of GMSK. The packet drop for different scenarios at different data rates are also estimated. To visualize the performance of handover, we estimated the loss percentage of packets when either VLC link goes down or restores. We transmitted the UDP packets for 60 seconds, the performance of the system is measured using Linux IPERF utility which is specially designed to measure the performance among different platforms. A stream is transmitted between server and client and IPERF measures the performance in term of packet. We interrupted the VLC uplink several times by physically obstructing the optical link between receiver and LED source and obtained a packet loss of $9 \%$. We estimated packet loss for different data rates and time-bandwidth values, the packet loss was close to $9 \%$ while when we broke the uplink the packet drop was quite low i.e., 0.1-0.2. 
2

3

4

5

6

TABLE 3 Packet loss due to network switching.

\begin{tabular}{lccccc}
\hline & \multicolumn{2}{c}{ Bandwdith-Time (0.35) } & & Bandwdith-Time (0.5) & Bandwdith-Time (0.7) \\
\cline { 2 - 3 } \cline { 5 - 5 } & Bit rate 3.125 Mbps & Bit rate 6.255 Mbps & & Bit rate 6.255 Mbps & Bit rate 6.255 Mbps \\
\hline Downlink break & $9.4 \%$ & $9.0 \%$ & $9.0 \%$ & $9.0 \%$ \\
Downlink restore & $0.19 \%$ & $0.11 \%$ & $0.15 \%$ & $0.3 \%$ \\
Uplink break & $0.19 \%$ & $0.15 \%$ & $0.25 \%$ & $0.2 \%$ \\
Uplink restore & $0.15 \%$ & $0.15 \%$ & & $0.15 \%$ & $0.15 \%$ \\
\hline
\end{tabular}

Figure 9 shows the calculated SNR from (10) at different points in a $1 \mathrm{~m}$ x $1 \mathrm{~m}$ area, for numerous data rates. The SNR decreases with an increasing receiver angle as we move from the direct straight line between source and receiver. For calculation, we took receiver angle between 0 to 30 degree. The results show that SNR decreases for higher data rate, for an optimal SNR at the higher data rates the receiver angle must be within 30 degree [34]. Another important aspect is that the calculated SNR value increases as the distance between source and receiver is reduced, as shown in Figure 9. Figure 10 and Figure 11 show the interpacket delay and throughput of the system. From Figure 10, we can observe that the inter-packet delay is negligible, and the system can send packets at different data rates without any additional delay unless the active communication link is disturbed. Figure 11 shows that the system is capable of transmitting data at different rates and the packet loss is negligible unless the VLC link is interrupted. The straight lines obtained in both figures show that system transmits packets without any loss and delay as long as the default VLC link is un-interrupted. The packets loss occurs when the interruption occurs, as can be seen in Table 3.

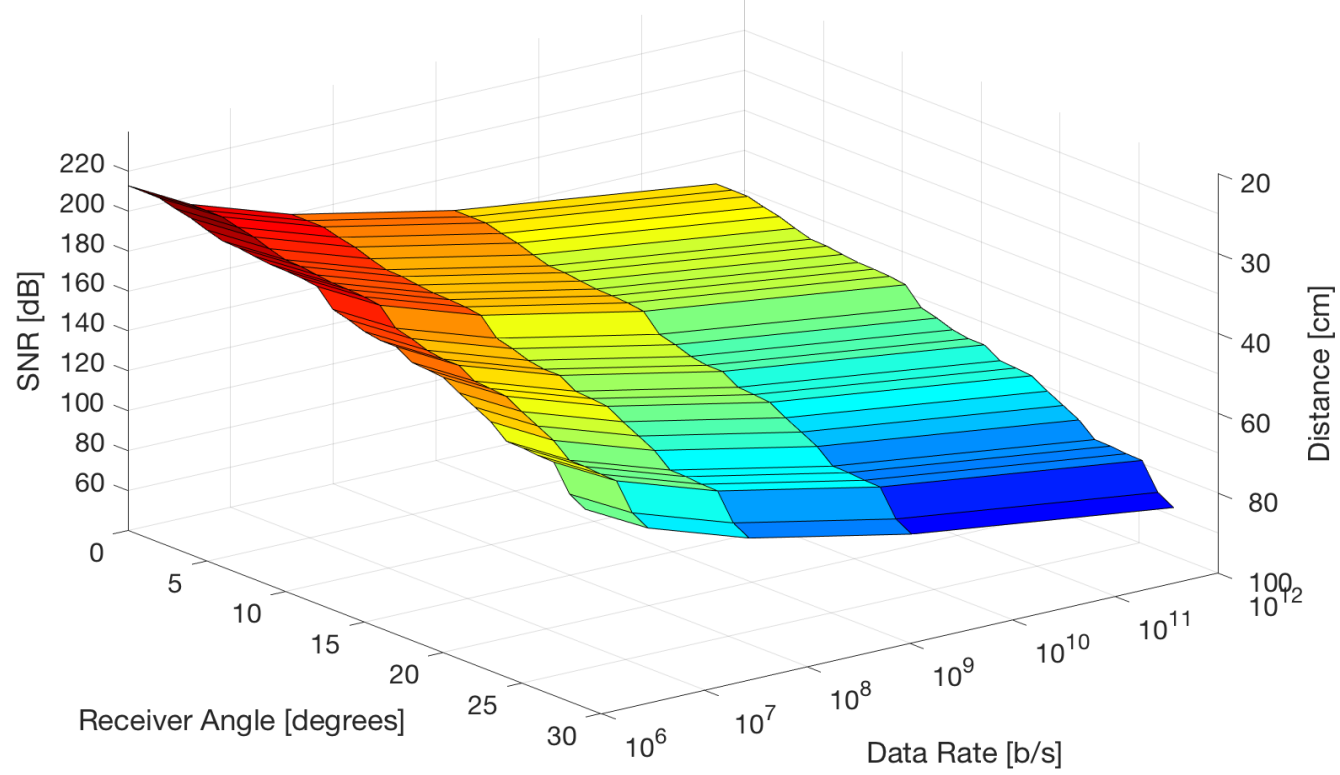

FIGURE 9 Theoretically achieved SNR vs data rate. 


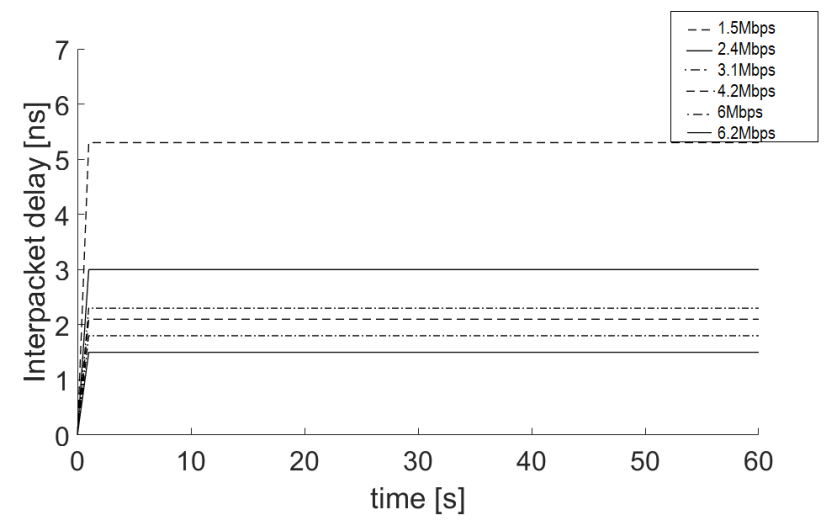

FIGURE 10 Interpacket delay; delay is plotted along y axis. The maximum delay between packets is $5 \mathrm{~ns}$.



FIGURE 11 System Througput; different data rates have been observed for certain time, the maximum data rate we observed is $6.2 \mathrm{Mbits} / \mathrm{s}$.

\section{4 | CONCLUSIONS}

In this paper, we introduced the concept of dynamically reconfigurable optical-radio wireless networks. A switching algorithm selects dynamically one operating mode out of a number of possible modes. Moreover, we demonstrated such a system by implementing and evaluating a simple reconfigurable hybrid network. The implemented system is based on conventional offthe-shelf components and the use of USRP modules for tunneling. USRPs allow to easily implement a dynamically configurable network. GNU radio software was used for packet encapsulation.

The operating modes have been designed to adapt the wireless networks to the dynamics of the channels, local operating policies, type of environments and others. In the practical implementation, a system maximizing the use of VLC links was considered, aiming at environments that are sensitive to interference and radio exposure. In practice the system switches from modes according to the switching algorithm. Clearly, there is some affinity between modes and certain scenarios. Mode 1 (fully optical) is mostly preferred in radio-sensitive environments, Hybrid Modes 2 and 3 can be selected upon link disruption, or depending on resource availability, for instance. Mode 4, fully radio, exploits all the advantages of radio such as better coverage, and can also be used when no light is available (e.g., nighttime). Mode 5 (optical and radio) can be used when larger capacity or enhanced reliability are required.

Video was streamed using UDP between source and sink nodes and we estimated the performance of the designed system in term of packet loss when transmitting the data i.e., video between two nodes. Seamless data transmission is of utmost importance for the hybrid system because the network switching could occur frequently due to the breakdown in the optical link and results in communications loss. In order to measure the performance of VHO we broke the optical link physically, number of times and the estimated packet loss shows that our system works well on UDP. GMSK modulation was used in the current implementation, though other more advanced schemes such as OFDM and color-shift keying will be implemented in the future to achieve higher data throughputs.

The current system only supports single-user vertical handover (switching modes) but in future we plan to extend the system to consider multi-user support as well as horizontal handovers between multiple VLC hotspots. Also, the switching algorithm will be further studied, aiming at better switching decisions, considering a number of relevant input parameters as well as exploiting machine learning techniques. Another important future contribution can be enhancing the adaptation capabilities of the system. This could be done by extracting affecting features from environment and letting the system to train itself on available features. In this way system would become more powerful to decide and switch according to the current needs.

The discussed concept of dynamically reconfigurable optical-radio wireless networks is a highly flexible high-performance approach to wireless networking. We expect that such hybrid wireless networks will become part of 5G and beyond. In addition of the aforementioned flexibility and performance, these networks can use the available radio resources much more efficiently. The flexibility of the concept also greatly suits the stringent requirements of on-demand wireless networks, a highly promising paradigm of future wireless communications. 


\section{References}

1. Jungnickel V., Uysal M., Serafimovski N., Baykas T., O’Brien D., Ciaramella E., Ghassemlooy Z., Green R., Haas H., Haigh P.A. et al. (2015) A european view on the next generation optical wireless communication standard. IEEE Conference on Standards for Communications and Networking (CSCN), pp. 106-111.

2. Kahn J.M. \& Barry J.R. (1997) Wireless infrared communications. Proceedings of the IEEE 85, pp. 265-298.

3. O’Brien D. \& Katz M. (2005) Optical wireless communications within fourth-generation wireless systems. Journal of optical networking 4, pp. 312-322.

4. Perez-Jimenez R., Rufo J., Quintana C., Rabadan J. \& Lopez-Hernandez F. (2011) Visible light communication systems for passenger in-flight data networking. IEEE International Conference on Consumer Electronics (ICCE), pp. 445-446.

5. Murai R., Sakai T., Kawano H., Matsukawa Y., Kitano Y., Honda Y. \& Campbell K.C. (2012) A novel visible light communication system for enhanced control of autonomous delivery robots in a hospital. IEEE/SICE International Symposium on System Integration (SII), pp. 510-516.

6. Hou J. \& O'Brien D.C. (2006) Vertical handover-decision-making algorithm using fuzzy logic for the integrated radio-andow system. IEEE Transactions on Wireless Communications 5, pp. 176-185.

7. Kim D.H., Kim W.T., Lee H.G., Kim S.J. \& Lee C.H. (2008) A performance evaluation of vertical hanover architecture with low latency handover. In: Convergence and Hybrid Information Technology, 2008. ICHIT'08. International Conference on, IEEE, pp. 66-69.

8. Ding W., Yang F., Yang H., Wang J., Wang X., Zhang X. \& Song J. (2015) A hybrid power line and visible light communication system for indoor hospital applications. Computers in Industry 68, pp. 170-178.

9. Rahaim M., Miravakili A., Ray S., Koomson V., Hella M. \& Little T. (2014) Software defined visible light communication. Wireless Innovation Forum Conference on Communications Technologies and Software Defined Radio (WInnComm SDR), pp. 11-13.

10. Zeng L., O’Brien D.C., Le Minh H., Faulkner G.E., Lee K., Jung D., Oh Y. \& Won E.T. (2009) High data rate multiple input multiple output (mimo) optical wireless communications using white led lighting. IEEE Journal on Selected Areas in Communications 27.

11. Wang Y., Chi N., Wang Y., Tao L. \& Shi J. (2015) Network architecture of a high-speed visible light communication local area network. IEEE Photonics Technology Letters 27, pp. 197-200.

12. Tiwari S.V., Sewaiwar A. \& Chung Y.H. (2017) Smart home multi-device bidirectional visible light communication. Photonic Network Communications 33, pp. 52-59.

13. Chowdhury H. \& Katz M. (2014) Cooperative data download on the move in indoor hybrid (radio-optical) wlan-vlc hotspot coverage. Transactions on Emerging Telecommunications Technologies 25, pp. 666-677.

14. Bao X., Zhu X., Song T. \& Ou Y. (2014) Protocol design and capacity analysis in hybrid network of visible light communication and ofdma systems. IEEE transactions on vehicular technology 63, pp. 1770-1778.

15. Rahaim M.B., Vegni A.M. \& Little T.D. (2011) A hybrid radio frequency and broadcast visible light communication system. IEEE GLOBECOM Workshops (GC Wkshps), pp. 792-796.

16. Saud M.S., Chowdhury H. \& Katz M. (2017) Heterogeneous software-defined networks: Implementation of a hybrid radiooptical wireless network. In: IEEE Wireless Communications and Networking Conference (WCNC), pp. 1-6.

17. Saud M.S. \& Katz M. (2017) Implementation of a hybrid optical-rf wireless network with fast network handover. 23th European Wireless Conference; Proceedings of European Wireless, pp. 1-6.

18. Simon M.K. (2005) Bandwidth-efficient digital modulation with application to deep-space communications, vol. 2. John Wiley \& Sons. 
19. Oller J., Garcia E., Lopez E., Demirkol I., Casademont J., Paradells J., Gamm U. \& Reindl L. (2014) Ieee 802.11-enabled wake-up radio system: Design and performance evaluation. Electronics Letters 50, pp. 1484-1486.

20. Philips myliving ceiling light 69067/31/16. accessed 02.02.2018 [online]. available: https://www.p4c.philips.com/cgi-bin/ cpindex.pl?scy=GB\&slg=EN\&ctn=690673116/

21. Le Minh H., O’Brien D., Faulkner G., Zeng L., Lee K., Jung D. \& Oh Y. (2008) High-speed visible light communications using multiple-resonant equalization. IEEE Photonics Technology Letters 20, pp. 1243-1245.

22. Khalid A., Cossu G., Corsini R., Choudhury P. \& Ciaramella E. (2012) 1-gb/s transmission over a phosphorescent white led by using rate-adaptive discrete multitone modulation. IEEE Photonics Journal 4, pp. 1465-1473.

23. Abirami M., Hariharan V., Sruthi M., Gandhiraj R. \& Soman K. (2013) Exploiting gnu radio and usrp: an economical test bed for real time communication systems. Fourth International Conference on Computing, Communications and Networking Technologies (ICCCNT), pp. 1-6.

24. Murota K. \& Hirade K. (1981) Gmsk modulation for digital mobile radio telephony. IEEE Transactions on communications 29, pp. 1044-1050.

25. Proakis J.G. (1995), Digital communications.

26. Sundberg C., Anderson J. \& Aulin T. (1986), Digital phase modulation. plenum press, new york and london.

27. Bohm B., Schoonees J. \& Braun R. (1994) Data-to-frequency mappings in various msk schemes. Electronics \& communication engineering journal 6, pp. 13-20.

28. Xiong F. (2000), Digital modulation techniques. norwood, ma, usa: Artech house.

29. Yu Chen Zhongyu Li P.L. \& Liu S. (2017) The ber performance comparison of msk and gmsk schemes for short-range visible light communication. IEEE 9th International Conference on Communication Software and Networks (ICCSN), pp. 611-614.

30. Linux kernel documentation. accessed 02.02.2018 [online]. available: https://www.kernel.org/doc/Documentation/

31. Gnu radio. accessed 02.02.2018 [online]. available:https:/gnuradio.org/redmine/projects/gnuradio/

32. Nguyen H., Choi J.H., Kang M., Ghassemlooy Z., Kim D., Lim S.K., Kang T.G. \& Lee C.G. (2010) A matlab-based simulation program for indoor visible light communication system. In: Communication Systems Networks and Digital Signal Processing (CSNDSP), 2010 7th International Symposium on, IEEE, pp. 537-541.

33. Chun H., Chiang C.J. \& O’Brien D.C. (2012) Visible light communication using oleds: Illumination and channel modeling. In: Optical Wireless Communications (IWOW), 2012 International Workshop on, IEEE, pp. 1-3.

34. Komine T. \& Nakagawa M. (2004) Fundamental analysis for visible-light communication system using led lights. IEEE transactions on Consumer Electronics 50, pp. 100-107.

\section{Acknowledgement}

Authors would like to thank Academy of Finland's support in the project HERONET, where this research work was mainly carried out. In addition, the support from Academy of Finland 6Genesis Flagship Project is warmly acknowledged. 









$389 \times 260 \mathrm{~mm}(300 \times 300 \mathrm{DPI})$ 


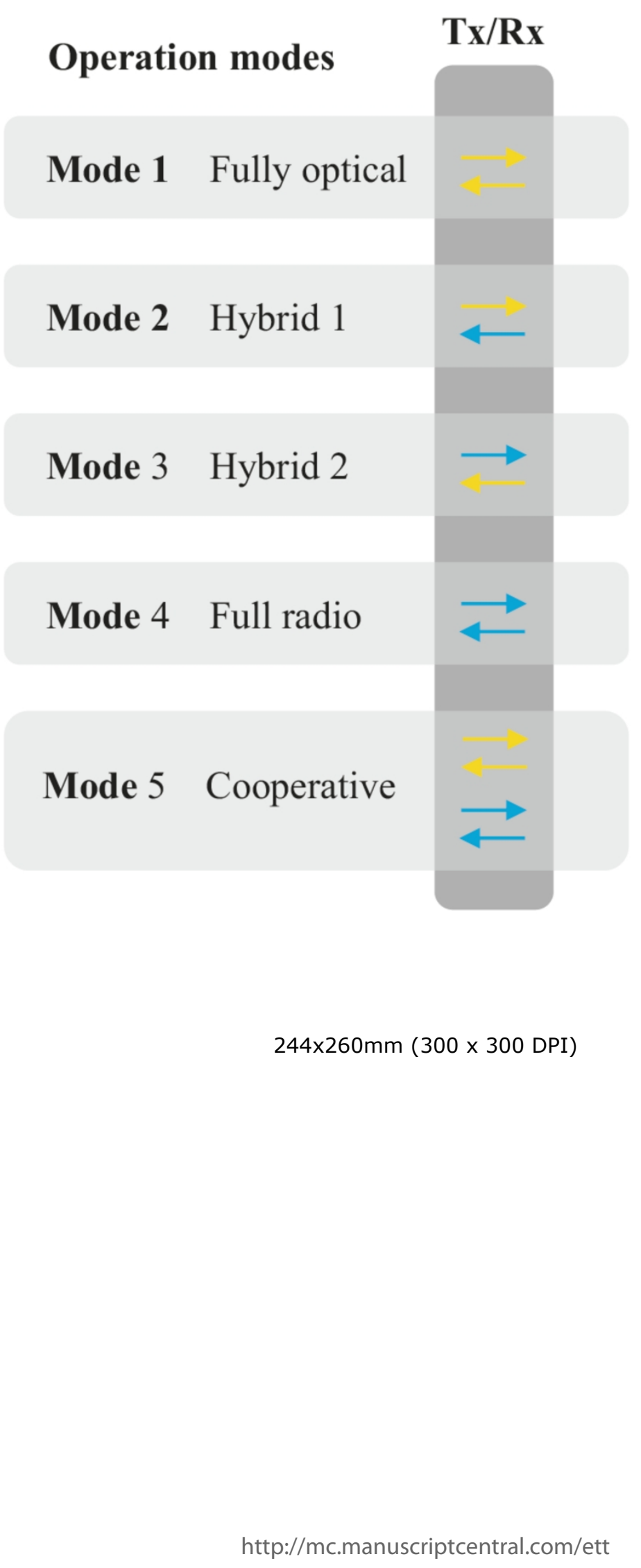




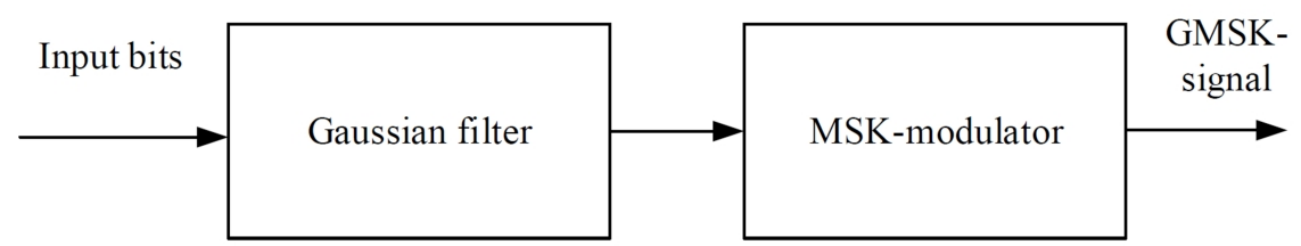

$461 \times 95 \mathrm{~mm}(300 \times 300$ DPI $)$ 




$75 \times 141 \mathrm{~mm}(300 \times 300$ DPI $)$ 


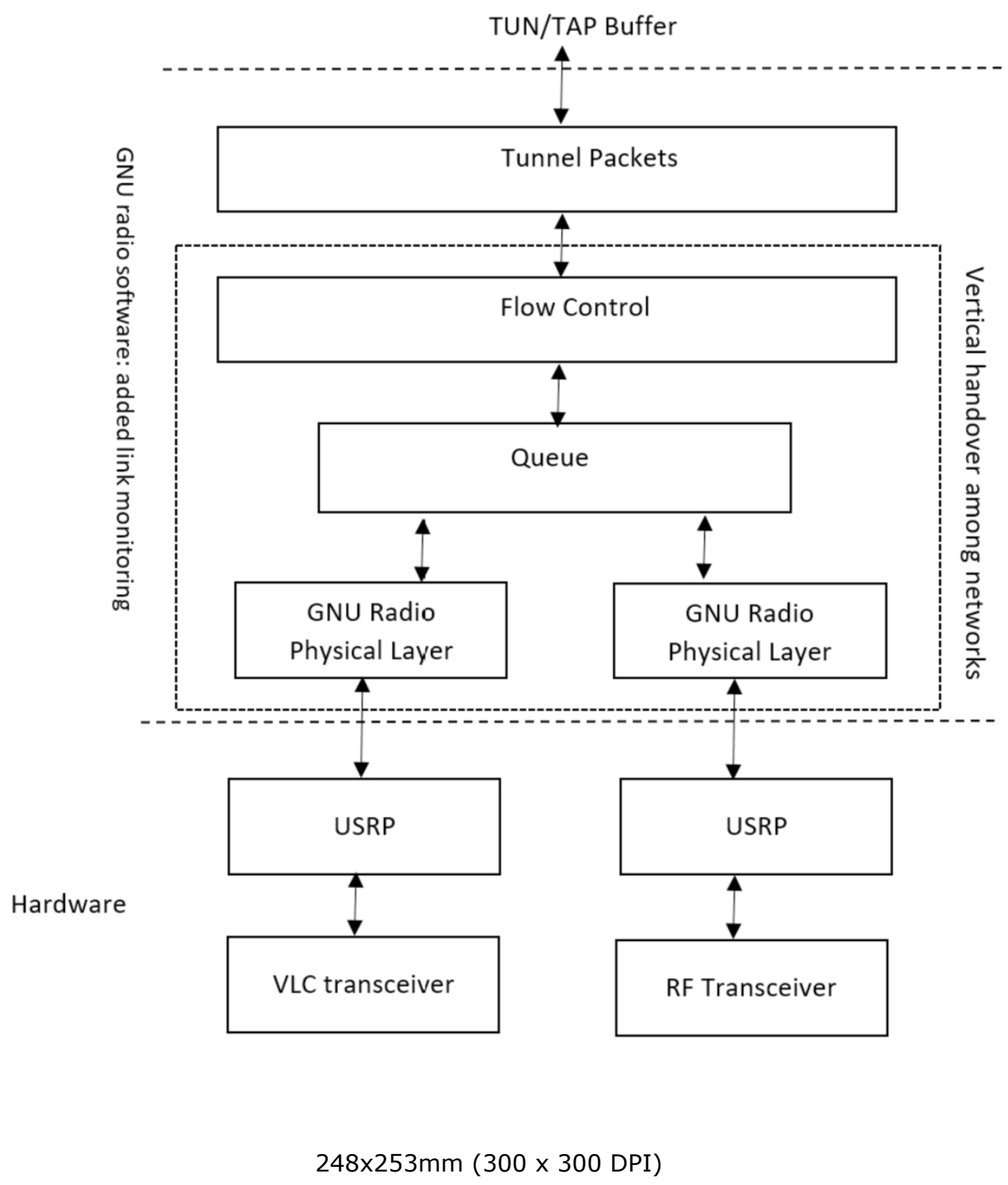






$296 \times 222 \mathrm{~mm}(300 \times 300 \mathrm{DPI})$

http://mc.manuscriptcentral.com/ett 


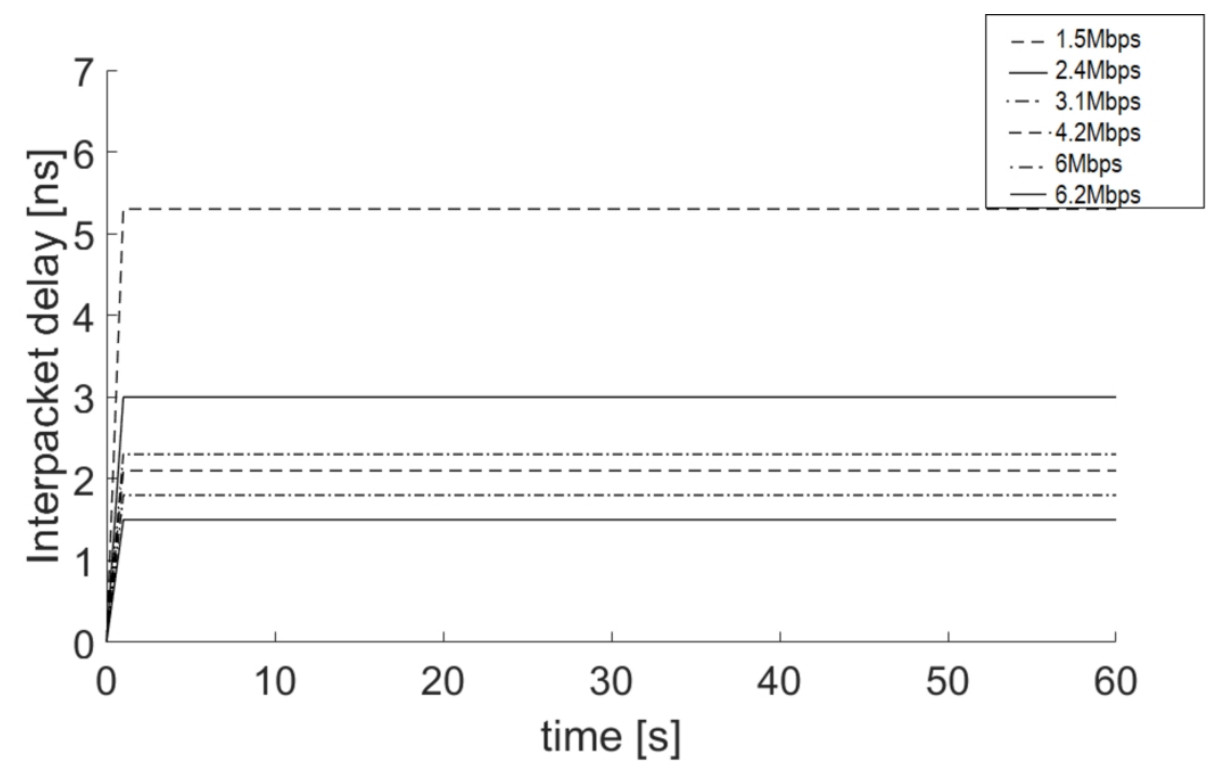

$276 \times 158 \mathrm{~mm}(300 \times 300 \mathrm{DPI})$ 


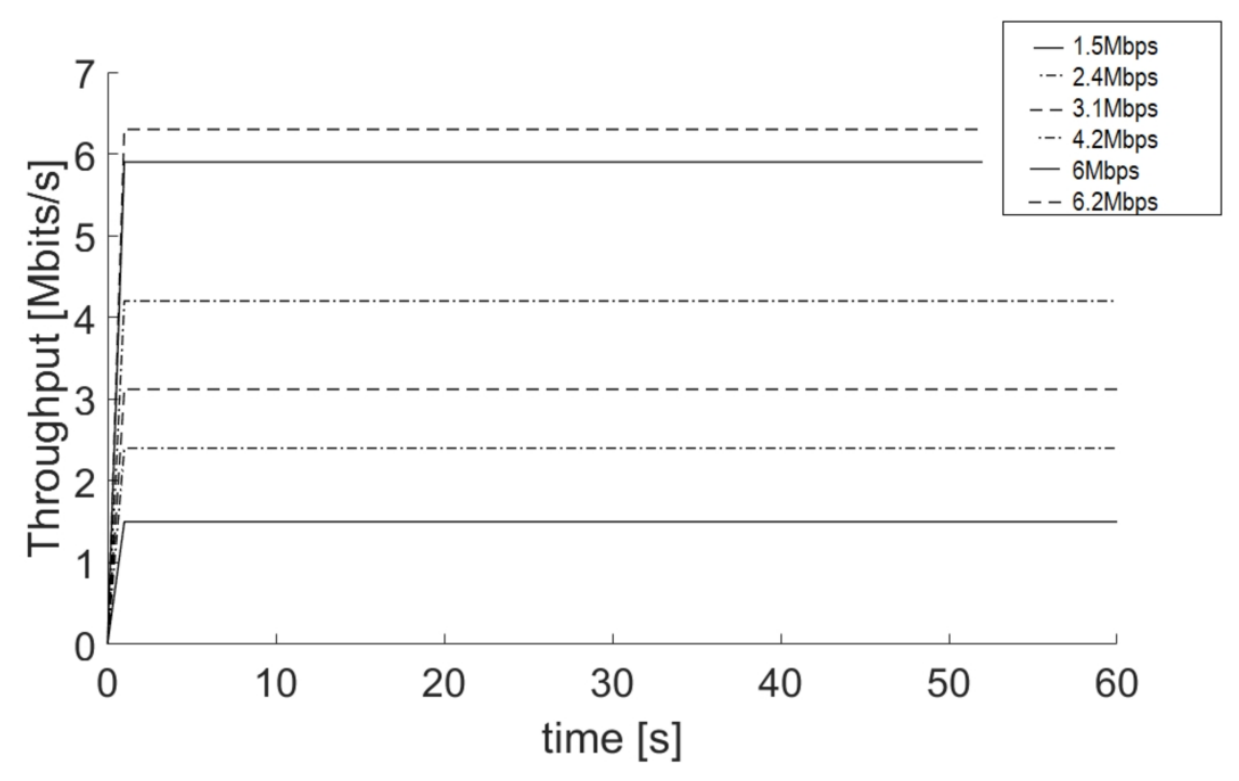

$276 \times 158 \mathrm{~mm}(300 \times 300$ DPI $)$ 


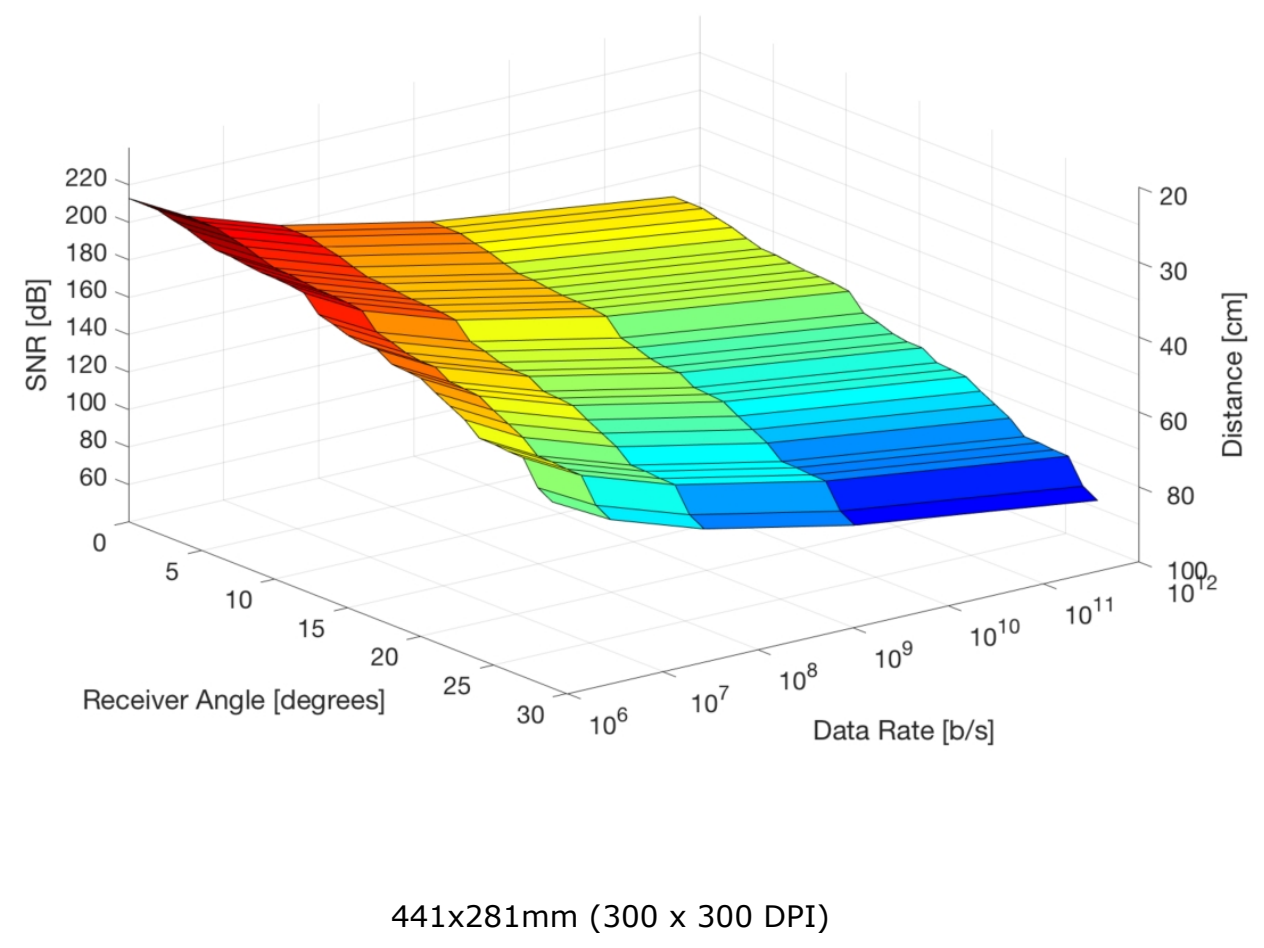

